\title{
Optical Laser-Induced CO Desorption from $R u(0001)$ Monitored with a Free-Electron X-ray Laser: DFT Prediction and X-ray Confirmation of a Precursor State
}

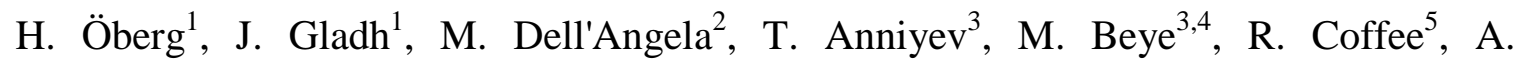
Föhlisch $^{4,6}$, T. Katayama ${ }^{3}$, S. Kaya ${ }^{3}$, J. LaRue ${ }^{3}$, A. Møgelhøj ${ }^{7,8}$, D. Nordlund ${ }^{9}$, H. Ogasawara $^{9}$, W. F. Schlotter ${ }^{5}$, J. A. Sellberg ${ }^{1,3}$, F. Sorgenfrei ${ }^{2}$, J. J. Turner ${ }^{5}$, M. Wolf ${ }^{11}$, W. Wurth ${ }^{2}$, H. Öström ${ }^{1}$, A. Nilsson ${ }^{1,3,7,9}$, J. K. Nørskov ${ }^{7,10}$, L. G. M. Pettersson ${ }^{1 *}$

${ }^{1}$ Department of Physics, AlbaNova University Center, Stockholm University, SE-10691, Sweden

${ }^{2}$ University of Hamburg and Center for Free Electron Laser Science, Luruper Chausse 149, D-22761 Hamburg, Germany

${ }^{3}$ SIMES, SLAC National Accelerator Laboratory, 2575 Sand Hill Road, Menlo Park, CA 94025, USA

${ }^{4}$ Institute for Methods and Instrumentation in Synchrotron Radiation Research, Helmholtz-Zentrum Berlin für Materialien und Energie GmbH,Wilhelm-Conrad-Röntgen Campus, Albert-Einstein-Str. 15, 12489 Berlin, Germany

${ }^{5}$ Linac Coherent Light Source, SLAC National Accelerator Laboratory, 2575 Sand Hill Road, Menlo Park, CA 94025, USA

${ }^{6}$ Institut für Physik und Astronomie, Universität Potsdam, Karl-Liebknecht-Str. 24-25, 14476 Potsdam, Germany

${ }^{7}$ SUNCAT Ctr Interface Sci \& Catalysis, SLAC National Accelerator Laboratory, 2575 Sand Hill Road, Menlo Park, CA 94025, USA

${ }^{8}$ CAMD, Department of Physics, Technical University of Denmark, DK 2800 Lyngby, Denmark

${ }^{9}$ Stanford Synchrotron Radiation Lightsource, SLAC National Accelerator Laboratory, 2575 Sand Hill Road, Menlo Park, CA 94025, USA

${ }^{10}$ SUNCAT Ctr Interface Sci \& Catalysis, Department of Chemical Engineering, Stanford University, Stanford, CA 95305, USA 
${ }^{11}$ Fritz-Haber Institute of the Max-Planck-Society, Faradayweg 4-6, D-14195 Berlin, Germany

*Correspondence to: 1gm@fysik.su.se

\begin{abstract}
We present density functional theory modeling of time-resolved optical pump/x-ray spectroscopic probe data of $\mathrm{CO}$ desorption from $\mathrm{Ru}(0001)$. The BEEF van der Waals functional predicts a weakly bound state as precursor to desorption. The optical pump leads to a near-instantaneous ( $<100 \mathrm{fs}$ ) increase of the electronic temperature to nearly $7000 \mathrm{~K}$. The temperature evolution and energy transfer between electrons, substrate phonons and adsorbate is described by the two-temperature model and found to equilibrate on a timescale of a few picoseconds to an elevated local temperature of 2000 K. Estimating the free energy based on the computed potential of mean force along the desorption path, we find an entropic barrier to desorption (and by time-reversal also to adsorption). This entropic barrier separates the chemisorbed and precursor state, and becomes significant at the elevated temperature of the experiment $(\sim 1.4 \mathrm{eV}$ at $2000 \mathrm{~K})$. Experimental pump-probe x-ray absorption/x-ray emission spectroscopy indicate population of a precursor state to desorption upon laser-excitation of the system [Dell'Angela et al., Science 339, 1302 (2013)]. Computing spectra along the desorption path confirms the picture of a weakly bound transient state arising from ultrafast heating of the metal substrate.
\end{abstract}

Keywords: CO desorption, potential of mean force, two-temperature model, pumpprobe, $\mathrm{x}$-ray spectroscopy, density functional theory 


\section{Introduction}

$\mathrm{CO}$ bonding to a metal surface is a prototypical process relevant to a large number of important chemical transformations, such as, e.g., the water gas shift reaction converting $\mathrm{CO}$ and $\mathrm{H}_{2} \mathrm{O}$ to $\mathrm{CO}_{2}$ and $\mathrm{H}_{2}$, the Fischer-Tropsch process to synthesize alkanes as synthetic fuels, as well as in conversion of $\mathrm{CO}$ to $\mathrm{CO}_{2}$ in car emission control catalysts. The adsorption, bonding and desorption of $\mathrm{CO}$ at a metal catalyst surface thus become of fundamental importance to understand in detail.

The static $\mathrm{CO}$ bond to a metal surface has been studied by a large number of authors, both experimentally, e.g., refs.[1-5], and theoretically, e.g., refs.[6-16]. The bonding is popularly described in terms of $\sigma$-donation and $\pi^{*}$ back-donation in a frontier orbital scheme, but detailed experimental measurements using $\mathrm{x}$-ray emission spectroscopy (XES) in combination with density functional theory (DFT) spectrum simulations have revealed a different picture. The bonding is found to occur in the $\pi$ system where the three interacting orbitals $\left(1 \pi, 2 \pi^{*}\right.$ and metal $\left.3 d_{\pi}\right)$ hybridize to form totally bonding $(1 \widetilde{\boldsymbol{\pi}})$, non-bonding $\left(\widetilde{\boldsymbol{d}}_{\boldsymbol{\pi}}\right)$ and totally antibonding $\left(2 \widetilde{\boldsymbol{\pi}}^{*}\right)$ states in an allylic configuration $[1 ; 2 ; 4-6 ; 16 ; 17]$; the tilde $(\sim)$ indicates that the orbital has been modified through the metal-adsorbate interaction. The closed-shell $5 \sigma$ orbital, on the other hand, generates exchange repulsion against the $d$-states of the metal $[1 ; 2 ; 4 ; 10 ; 17]$. We note here that the $\pi$-bonding in terms of the allylic model was described already by Blyholder [6] using extended Hückel theory while the $\sigma$-repulsion became most evident from experimental XES studies of CO adsorption in different sites on $\mathrm{Ni}(100)$ [2].

Here we will expand on previous reports $[18 ; 19]$ on the time-resolved electronic structure changes in $\mathrm{CO}$ desorption from a transition-metal surface, $\mathrm{Ru}(0001)$, studied using the Linac Coherent Light Source (LCLS) free-electron x-ray laser. We will, in particular, focus on the interplay between theory and experiment in the analysis of timeresolved XES and x-ray absorption spectroscopy (XAS) data measured subsequent to heating the substrate with an optical pump laser. DFT calculations of the temperaturedependent free-energy potential energy surface were performed and XE and XA spectra calculated along the path to desorption. Through this interplay between experiment and theory we could unambiguously spectroscopically identify the proposed precursor state to desorption and adsorption [18; 19]. 


\section{Methods}

\section{Computational methods}

The $\mathrm{Ru}(0001)$ surface was modeled as periodically repeated three-layer slabs of which the bottom two layers were kept fixed during structure relaxation (unit cell given in the Supplementary Material). Increasing the slab with one layer leads to no significant difference in the simulated spectra and the variations in $\mathrm{CO}$ adsorption energy were deemed negligible for the results in the present study. A $2 \times 2$ cell with periodic boundary conditions was used in which a CO molecule was adsorbed at the ontop site, hence a coverage of 0.25 ML. From the top of the $\mathrm{CO}$ molecule to the bottom of the adjacent slab a separation of $10 \AA$ of vacuum was employed in the surface normal direction. The DFT calculations of the potential energy surface of $\mathrm{CO}$ desorption were performed using the BEEF-vdW exchange-correlation (XC) functional [20] as implemented in the grid-based real-space projector-augmented wave GPAW code [21; 22]. BEEF-vdW includes van der Waals (vdW) interactions through a functional describing non-local correlation; to establish the effects of including the $\mathrm{vdW}$ interaction the generalized gradient approximation (GGA) XC functional RPBE [23] was also applied. A grid-spacing of 0.18 $\AA$, and $0.12 \mathrm{eV}$ Fermi smearing were used in the calculations. To converge the adsorption energy as well as the simulated spectra, a $k$-point mesh of $4 \times 4 \times 1$ was found to be sufficient. In determining the minimum energy path (MEP) $0.05 \mathrm{eV} / \AA$ was used as acceptance criterion on the maximum force on each relaxed atom; tightening the criterion to $0.02 \mathrm{eV} / \mathrm{A}$ only changes the total energy of chemisorbed $\mathrm{CO}$ by $0.1 \mathrm{meV}$.

O K-edge XE spectra were calculated using GPAW [24] from the ground state orbitals [25] and summed over $\mathrm{p}_{\mathrm{xy}}$ and $\mathrm{p}_{\mathrm{z}}$ symmetries; the RPBE functional was used in this case. To compare with the experimental spectra a broadening with a Gaussian function of $1 \mathrm{eV}$ full-width at half-maximum (FWHM) was employed. Geometries of the chemisorbed state and the precursor state were taken from the MEP of desorption and to obtain an absolute energy scale the computed spectra were shifted so that the energy position of the $1 \tilde{\pi}$ state is aligned with that of the experiment; note that in the experimental spectrum the deconvolved $1 \tilde{\pi}$ peak remains fixed in energy. 
O K-edge XA spectra and core-excited state wave functions were obtained using the StoBe-deMon [26] DFT code using a three-layer cluster model containing 17 Ruthenium atoms to represent the $\mathrm{Ru}(0001)$ surface (coordinates given in the Supplementary Material). Triple-zeta basis sets were used in combination with a 14 electron relativistic effective core potential for Ruthenium, while carbon was described by an all-electron triple-zeta basis with one $d$-function added. Oxygen was described by the IGLO III basis of Kutzelnigg and coworkers [27] to allow for relaxation of the core after removal of one $1 s$ electron. Spectra were computed within the transition potential approach of Triguero et al. [28; 29]. A Gaussian broadening of $0.5 \mathrm{eV}$ FWHM was applied up to $534 \mathrm{eV}$ and then linearly increased to $3 \mathrm{eV}$ until $539 \mathrm{eV}$ and constant beyond that. The energy scale was adjusted by shifting the spectrum at the chemisorbed equilibrium geometry to have its $2 \tilde{\pi}^{*}$ peak aligned with that of the unpumped experiment; the same shift was then applied to all other spectra. The XAS calculations used the exchange by Becke [30] and the correlation functional by Perdew [31]; it has been shown [32] that the spectral shape does not depend significantly on the choice of functional.

\section{Experimental setup and method}

The optical pump/x-ray probe experiment was conducted at the Surface Chemistry Endstation of the soft x-ray materials science instrument (SXR) [33-35] at the LCLS facility [36]. The preparation of the sample and the details about the experimental procedure can be found elsewhere $[18 ; 19]$.

\section{Results}

\section{Minimum energy path}

In Fig. 1 we present the computed minimum potential energy surface (corresponding to 0 $\mathrm{K})$ along the desorption coordinate of $\mathrm{CO}$ along the surface normal from the ontop site. Depending on the choice of $\mathrm{XC}$ functional, i.e. if non-local correlation is included to describe vdW interactions or not, the long-range behavior differs significantly. As seen in Fig. 1, RPBE actually produces a small barrier at a CO-Ru distance of $\sim 3.1 \AA$ after which the potential energy trails off to zero. The BEEF-vdW functional, on the other hand, yields an attractive plateau starting at a C-Ru distance around $3 \AA$ which can be 
populated in the desorption process. This long-range attraction is absent when using standard GGA functionals such as RPBE. The equilibrium $\mathrm{Ru}-\mathrm{C}$ distance for the favored ontop adsorption was obtained as $1.94 \AA$.

As seen from the inset in Fig. 1, at distances beyond $\sim 4 \AA$ the $\mathrm{CO}$ molecule becomes free to rotate in the shallow vdW-generated shelf which gives a significant contribution to the entropy in the desorption process, a discussion we will return to below. At distances smaller than $2.5 \AA$ in the figure, $\mathrm{CO}$ is found to move from ontop to bridge and hollow sites.

To simulate the desorption process at conditions relevant to the experiment we need to account for the temperature of the system after laser excitation and, in addition, include the entropic contribution to obtain the finite temperature potential-energy surface through the potential of mean force (PMF). In the standard approach, the energy transfer from the laser beam to the metal substrate is described using the two-temperature model (2TM) of Anisimov et al. [37].

\section{Two-Temperature Model (2TM)}

The optical laser pulses ( $\lambda=400 \mathrm{~nm}$ in this experiment) that are used to initiate the reaction are absorbed by the metal substrate causing excitation of substrate electrons that thermalize on a time-scale of $\sim 100$ fs [38], after which a temperature can be defined for the electrons. The hot electrons subsequently heat up the lattice via electron-phonon coupling and may also couple to the adsorbate vibrational degrees of freedom. Finally, the system equilibrates to an elevated temperature. The time-dependent temperatures of the electrons $\left(\mathrm{T}_{e l}\right)$ and phonons $\left(\mathrm{T}_{p h}\right)$ can be modeled, within the limit of thermalization, with the 2TM [37] where the electron and phonon systems are treated as two coupled heat baths where we use the same formalism and material parameters as in ref. [39]:

$$
\begin{aligned}
& C_{e l} \frac{\partial}{\partial t} T_{e l}=\nabla\left(\kappa \nabla T_{e l}\right)-g\left(T_{e l}-T_{p h}\right)+S(z, t) \\
& C_{p h} \frac{\partial}{\partial t} T_{p h}=g\left(T_{e l}-T_{p h}\right)
\end{aligned}
$$

The heat capacity of the electron system is $C_{e l}=\gamma T_{e l}$ with electron specific heat $\gamma=400 \mathrm{~J} / \mathrm{m}^{3} \mathrm{~K}^{2}$ while the heat capacity $C_{p h}$ of the phonon system is given by the Debye 
model. The thermal conductivity is $\kappa=\kappa_{0} T_{e l} / T_{p h}$ with $\kappa_{0}=117 \mathrm{~W} / \mathrm{Km}$ and the electron-phonon coupling is $g=1.85 \times 10^{18} \mathrm{~W} / \mathrm{m}^{3} \mathrm{~K}$. The laser excitation is given as a function of depth $z$ and time $t$ by $S(z, t)=I(t) \lambda^{-1} e^{-\frac{z}{\lambda}}$ where $I(t)$ is the time-dependent absorbed intensity of the laser pulse and $\lambda$ the penetration depth. Using the 2TM, we computed the electron and phonon temperatures assuming a Gaussian time-dependence with a FWHM of $50 \mathrm{fs}$ and an absorbed fluence of $140 \mathrm{~J} / \mathrm{m}^{2}$, which yields an electronphonon equilibration time of $1.3 \mathrm{ps}$ and a temperature at this time of $1850 \mathrm{~K}$. The modeled time-evolution of the electron and phonon temperatures at the surface during the first 14 ps is shown in Fig. 2.

The two-temperature model has been shown experimentally to significantly overestimate the resulting temperatures at low fluence due to efficient energy dissipation into the bulk via ballistic electrons [40]. As noted in the reference, the role of ballistic electrons is expected to decrease with increasing fluence through enhanced electronelectron coupling. We thus expect that the two-temperature model provides a much better description of the sample temperature in the present work with absorbed fluence $140 \mathrm{~J} / \mathrm{m}^{2}$ than was found in the low-fluence $\left(5.8 \mathrm{~J} / \mathrm{m}^{2}\right)$ experiments in reference [40].

Coupling the substrate sub-systems to the adsorbate system can be done using a friction model in which the energy flow from the substrate to the adsorbate vibrational degrees of freedom is described in terms of friction:

$$
\frac{d}{d t} T_{a d s}=\frac{1}{\tau_{e l}}\left(T_{e l}-T_{a d s}\right)+\frac{1}{\tau_{p h}}\left(T_{p h}-T_{a d s}\right)
$$

where $\tau_{e l}$ and $\tau_{p h}$ are the coupling constants of the electrons and phonons, respectively. Depending on the chemical reaction and substrate, the mechanism driving the reaction can differ significantly. For example, $\mathrm{CO}$ desorption from $\mathrm{Cu}$ can be described purely as an electron-mediated process [41], i.e. $\tau_{p h}=\infty$ in Eq. 2, while on Pd, friction coupling also to the phonon heat bath is required [42]. For $\mathrm{CO}$ desorption from $\mathrm{Ru}(0001)$ it has been proposed that coupling to both the electron and phonon heat baths is required to model the experiments accurately [43]. In that study, $\tau_{e l}=250 \mathrm{fs}$ and $\tau_{p h}=700 \mathrm{fs}$ was employed in the friction model. In Fig. 2 we display the adsorbate temperature of CO using these coupling constants and note a peak of the adsorbate temperature at $3450 \mathrm{~K}$ after which it 
equilibrates with the substrate subsystems at just below $2000 \mathrm{~K}$. With such elevated temperatures in the adsorbate system, entropy plays a significant role in the desorption process where transfer into the precursor state entails a loss of enthalpy but a significant gain in entropy.

\section{Potential of Mean Force}

We compute explicitly the potential of mean force (PMF) along the desorption coordinate to obtain the relevant finite temperature potential energy surface for the target temperatures of interest, i.e. temperatures of the magnitude modeled with the 2TM. We assume that the potential energy is separable in translational and rotational degrees of freedom

$$
V_{\text {tot }}=V_{0}+V_{x}+V_{y}+V_{\text {rot } 1}+V_{\text {rot } 2} \approx V_{0}+2 V_{\text {trans }}+V_{\text {rot } 1}+V_{\text {rot } 2}
$$

where the rotational potentials $\left(V_{r o t 1}\right.$ and $\left.V_{r o t 2}\right)$, translational potentials $\left(V_{x}\right.$ and $\left.V_{y}\right)$ and the potential of the MEP $\left(V_{0}\right)$ have been introduced. The high-frequency internal $\mathrm{CO}$ stretch vibration is neglected, since it is assumed to not be excited. On the right hand side of Eq. (3) it is also assumed that the two translational degrees of freedom on the surface are equivalent. As proposed by Doren and Tully [44; 45] the PMF, $W(s)$, can be written as

$$
W(s)=-k_{B} T \ln (g(s))+k_{B} T \ln (g(\infty)),
$$

where $k_{B}$ is Boltzmann's constant, $T$ is the temperature, $s$ is the distance from the surface to the center of mass of the $\mathrm{CO}$ molecule and $g(s)$ is given by the integral

$$
g(s)=\Gamma^{-1} \int e^{-\frac{V(s, \mathbf{q})}{k_{B} T}} d \mathbf{q}
$$

here $\mathbf{q}$ represents the four degrees of freedom (two rotations and two translations orthogonal to the path of desorption) and $\Gamma$ is an arbitrary normalization constant, the choice of which is irrelevant when the PMF is set to zero at infinite separation through equation (4).

Inserting Eq. (3) into Eq. (5) the following is obtained

$$
g(s)=\Gamma^{-1} \int e^{-\frac{V_{0}(s)+2 V_{\text {trans }}(s, x)+V_{\text {rot } 1}(s, \theta)+V_{\text {rot } 2}(s, v)}{\mathbf{k}_{\mathbf{B}} T}} d x d \theta d v=\Gamma^{-1} 2 g_{\text {trans }}(s) g_{\text {rot } 1}(s) g_{\text {rot } 2}(s)
$$


By adding and subtracting $V_{0}$ to each degree of freedom, the PMF can be written as a correction for each degree of freedom

$$
W_{t o t}(s)=V_{0}-\sum_{i}^{N}\left(W_{i}-V_{0, i}^{f i t}\right)
$$

where $V_{0}$ is the energy of the MEP (effectively the $z$-direction), $W_{\mathrm{i}}$ is the PMF and $V_{0, i}^{f i t}$ is the fit to $V_{0}$ for the i'th degree of freedom. $V_{0, i}^{\text {fit }}$ only differs from $V_{0}$ to the extent that our mathematical representation of the potential in this direction is approximate. By subtracting it out in each degree of freedom, this error, however, is cancelled out. Alternative, but more costly techniques that could have been used to obtain the free energy contribution include constrained molecular dynamics with, e.g., umbrella sampling. However, by constraining to specific, orthogonal degrees of freedom we can here directly compute the relevant potentials as described below.

To calculate the individual rotational contributions, the degrees of freedom were sampled by calculating the potential energy of the two rotations (cartwheel and helicopter; see Fig. 3) and a translation of the $\mathrm{CO}$ molecule at fixed surface distances using the BEEF-vdW XC functional. The potential energy curves for each degree of freedom were then fitted to functions for each surface distance and inserted in the integral in Eq. (6).

The potential energy of the translational motion was fitted to the function

$$
f 1(x)=a_{0}+a_{1} \cos (d \pi x)+a_{2} x^{8}
$$

where the periodicity $d$ in the cosines function is the distance from one ontop position to another. The translational potential was modeled using a $2 \times 4$ unit cell containing two $\mathrm{CO}$ molecules where one is fixed while the other is moved in the surface plane between the neighboring $\mathrm{CO}$ molecules at the same surface distance confining it and giving a "particle in a box"-like potential with a cosine bottom. We thus neglect changes to the translational potential from interacting molecules at different heights above the surface.

The fitting procedure for the translational motion was as follows: initially, all the coefficients were determined by fitting to all points. $a_{0}$ and $a_{2}$ were then fixed to the obtained values, while $a_{1}$ was refitted to the non-repulsive points (excluding the potential 
energy points where the $\mathrm{CO}$ molecules are too close to their neighbors). This was done in order to avoid destroying the description in the interesting low potential-energy region, which would be the case if the unimportant extremely high potential-energy values as the $\mathrm{CO}$ molecules get too close to their neighbors were included when fitting the cosines function.

The cartwheel and helicopter rotational modes were computed for a single $\mathrm{CO}$ in a $2 \times 2$ cell. The cartwheel rotation can be approximated with a cosine series

$$
f 3(x)=a_{0}+a_{1} \cos (x)+a_{2} \cos (2 x)+a_{3} \cos (3 x)+a_{4} \cos (4 x)+a_{5} \cos (5 x)
$$

while the helicopter rotational degree of freedom is simply given by

$$
f 4(x)=\frac{\Delta E}{2} \cos (6 v)+\frac{\Delta E}{2}+V_{0}
$$

where $v$ is the angle of periodicity, $V_{0}$ is the lowest potential energy and $\Delta E$ is the difference between the highest and lowest potential energy.

The helicopter rotation is not a normal mode when the $\mathrm{CO}$ molecule is close to the surface. As seen from the potential energy in the inset in Fig. 1, the parallel orientation of the $\mathrm{CO}$ molecule is very unstable close to the surface. In this region the cartwheel rotation is counted twice in the PMF. At $4 \AA$ surface distance and beyond, where the parallel CO orientation becomes as stable as the perpendicular, the contributions from both rotations are added.

In Fig. 4, we show, together with the MEP, the PMF of $\mathrm{CO}$ desorption from $\mathrm{Ru}(0001)$ at two temperatures and note a significant effect as entropy is included. Already at room temperature, two minima have developed: the chemisorption well and an outer, shallow well which corresponds to the precursor state to adsorption and desorption. These states are separated by a small entropic barrier which increases with temperature. At temperatures similar to those predicted by the 2TM, the barrier becomes significant; at $2000 \mathrm{~K}$, which is similar to the adsorbate temperature upon equilibrating with the substrate, it is $\sim 1.4 \mathrm{eV}$. The resulting barrier is a combination of contributions from enthalpy and entropy where, in desorption, enthalpy is lost more quickly than entropy is gained and the reverse in adsorption. We note that in other cases with less rapid loss of enthalpy, e.g., the coadsorption system $\mathrm{CO} / \mathrm{O} / \mathrm{Ru}(0001)$ [46], no entropic barrier develops in spite of the similarities with the $\mathrm{CO} / \mathrm{Ru}(0001)$ system studied in the present work. 
In the following, we will demonstrate experimental data supporting the theoretical predictions of a weakly bound precursor state further out from the surface.

\section{Experimental X-ray Spectroscopic Data}

Evidence of molecules populating the precursor state in the desorption process is found from time-dependent XES and XAS in conjunction with computed spectra and excited orbital spatial extents along the desorption path [18]. In this section we focus on the experimental data. For gas phase $\mathrm{CO}$, the resonant $\mathrm{O} 1 s \rightarrow 2 \pi^{*}$ excitation leads to a probability of the excited $2 \pi^{*}$ electron decaying back with emission of an x-ray photon of energy similar to the exciting photon (participator decay) since the electron remains strongly localized in this orbital during the core-hole lifetime. For chemisorbed CO the $2 \tilde{\pi}^{*}$ instead couples strongly with the conduction band and delocalizes, which eliminates the participator decay. In the precursor state, however, the $2 \pi^{*}$ still couples to the substrate, but weakly, leading to a specific signature of this state which we will return to below.

Fig. 5 shows measured XA and XE spectra for selected pump-probe delays. Details of how the spectra were obtained and normalized along with fitting procedures can be found elsewhere [18]. At negative time delays, i.e. before the laser pump excites the system, we probe the chemisorbed state of CO. After laser excitation, we observe significant changes in the spectra. In the XAS, a steady shift towards higher excitation energy is observed with increasing pump-probe delay together with an increase in $2 \tilde{\pi}^{*}$ peak intensity. Both these changes are consistent with a $\mathrm{CO}-\mathrm{Ru}$ bond weakening; as the CO-metal distance increases, the hybridization with the metal $d$ states becomes smaller and the width of the $2 \tilde{\pi}^{*}$ peak decreases as it moves towards, but not all the way to, the gas phase position of $\mathrm{CO}$.

Turning to the XES, we observe a number of changes in the spectra upon laserexcitation. The main peak containing the $5 \tilde{\sigma}$ and $1 \tilde{\pi}$ orbitals becomes narrower and shifts towards higher emission energy. In addition, a peak centered at $\sim 533 \mathrm{eV}$ is seen at pump-probe delay of +7 ps. To visualize the spectral changes, we plot in Fig. 6 the emission energy of the center of mass of the $5 \tilde{\sigma}$ and $1 \tilde{\pi}$ orbitals and the intensity of the 
$\tilde{d}_{\pi}$ state as functions of pump-probe delay. A decrease in $\tilde{d}_{\pi}$ intensity is observed which indicates a weakening of the CO-Ru interaction; the $\tilde{d}_{\pi}$ orbital typically has a lone-pair contribution on the $\mathrm{O}$ atom [47] which will diminish as the $\mathrm{CO}$ decouples from the surface. The $5 \tilde{\sigma} / 1 \tilde{\pi}$ peak shifts towards higher emission energy, also this demonstrating the appearance of a more weakly bound $\mathrm{CO}$ species.

We now turn our attention to the new peak arising around $533 \mathrm{eV}$. In Fig. 7, the oxygen K-edge XES spectra in the emission energy region corresponding to the elastically scattered photons are displayed. The contribution of the elastically scattered light to the fit of the XES spectra (elastic peak) is described by peak E with Gaussian lineshape and is kept constant in the fitting procedure at all pump-probe delays. However to fit the data an additional component (dark blue) with Gaussian lineshape (peak P) is required at $533.2 \mathrm{eV}$. The intensity of this peak increases as a function of pump-probe delay. This feature resembles the participator peak, i.e. the radiative decay of the coreexcited state to the ground state of the molecule, which has been observed in gas phase [48]. This decay channel is strongly suppressed for strongly interacting chemisorbed molecules due to ultrafast charge transfer out of the resonance into the metal substrate [49]. The presence of the decay channel and its increase as a function of pump-probe delay provide further support for the conclusion of laser-induced population of a more weakly bound state prior to desorption. In addition, the emission energy position of the participator peak of the precursor state is $\sim 0.5 \mathrm{eV}$ lower than that of gas phase $\mathrm{CO}$ [18].

To summarize, measured time-dependent XAS and XES data support the theoretical prediction of a precursor state to desorption for $\mathrm{CO} / \mathrm{Ru}(0001)$.

\section{Computed X-ray Spectroscopic Data}

The XAS and XES measurements indicate a precursor state signified by a shift to higher excitation energies and an intensity increase of the unoccupied $2 \tilde{\pi}^{*}$ resonance probed in the XAS. In addition, the changes in the XES after laser-excitation support the observation in XAS; a decrease in $\tilde{d}_{\pi}$ intensity along with small shift of the main peak, constituted by the $5 \tilde{\sigma}$ and $1 \tilde{\pi}$ states, to higher emission energies. Also, as the $\mathrm{CO}$ 
decouples from the surface and becomes more weakly bound a feature arises attributed to the participator decay.

In the following, computed XA and XE spectra for selected structures along the desorption path in Figs. 1 and 4 will be analyzed. Note that the theoretical approach employed to model XES considers only the non-resonant process so that effects of participator decay will not be captured in the computed spectra. Fig. 8 shows, along with the computed XA spectra, the core-excited $2 \tilde{\pi}^{*}$ wave function for a cluster model employed in the spectrum calculations. Four structures are sampled to simulate chosen sections of the desorption path: the equilibrium ontop structure of $\mathrm{CO}$ as well as three structures in the precursor well. We note that the core-excited state is fully delocalized at the equilibrium position while in the precursor state the delocalization depends on the distance. Turning to the spectra in e), the intensity in the $2 \pi^{*}$ peak at $533.5 \mathrm{eV}$ increases as the interaction with the substrate weakens and the peak position shifts to higher energy as the internal $\mathrm{CO}$ bond distance shortens in the precursor state. Comparing with experiment in Fig. 5 we find very similar effects with a shift to higher energy in the precursor state by $0.3 \mathrm{eV}$ in the calculations compared to $0.2 \mathrm{eV}$ in the experiment. The computed peak height increases by $\sim 40 \%$ compared to $\sim 30 \%$ in the measurement. Note that the $2 \pi^{*}$ core-excited state forms an anti-bonding combination with the metal states leading to a slightly lower energy for the farthest point where the anti-bonding character is less important.

In Fig. 1 we noted that the potential energy was independent of the orientation of the $\mathrm{CO}$ molecule in the precursor state. We therefore computed the spectra for CO parallel to the surface at the same distances as in Fig. 8 and show in Fig. 9 the core-excited $2 \pi^{*}$ orbital together with the spectra of the vertical and horizontal $\mathrm{CO}$ molecule in the precursor state. Looking at the wave function plots for the horizontal $\mathrm{CO}$, we note that at all points the core-excited state remains interacting with the substrate, but with weaker coupling at the longer distances. In the spectra, at the shortest distance to the surface, there is a small difference both in peak height and position between the vertical and horizontal species which diminishes with increasing COM distance in the precursor well, indicating that the distance dependence of the XA spectrum becomes independent of the orientation in the precursor state. 
In Fig. 10 we show the computed total and angular-resolved XE spectra for the chemisorbed and precursor states of $\mathrm{CO}$. Note that in the experiment the excitation is performed resonantly as $\mathrm{O} 1 s \rightarrow 2 \tilde{\pi}^{*}$. For chemisorbed $\mathrm{CO}$ the excited electron couples strongly to the substrate and delocalizes without affecting the subsequent emission process. In the precursor state the coupling to the substrate is weaker and the excited electron remains localized to a greater extent on the molecule and affects the decay. The non-resonantly computed spectrum is thus only representative of experiment for the chemisorbed state; particularly for the precursor state the excited electron should ideally be included but this is difficult to converge with the present techniques. Applying the full Kramers-Heisenberg formalism, including life-time vibrational interference effects, can be expected to broaden the computed spectra further [50], but in the present work we focus on the trends in the spectral peaks in a comparison of chemisorbed and precursor $\mathrm{CO}$.

In both cases the presented spectra give a correct projection of the $2 p$ character of the molecular orbitals onto the oxygen $1 s$, illustrating the formation of the new orbitals upon chemisorption and the orbital shifts and changes in character as the molecule moves into the precursor state. As the molecule moves to the precursor state we observe a shift of the $5 \tilde{\sigma}$ component towards higher energy and the chemisorption-induced $\tilde{d}_{\pi}$ state vanishes as the interaction with the substrate decreases. Compared to experiment in Fig. 5 the computed $5 \tilde{\sigma}$ state exhibits a similar loss of intensity due to rehybridization from more $\mathrm{O} 2 p$ lone-pair character in the chemisorbed state to more population on the carbon end as the molecule moves away from the substrate. Both in the experiment and the simulation the $4 \tilde{\sigma}$ state shows the opposite trend; this is expected as the exchange repulsion felt by the $5 \tilde{\sigma}$ against the substrate is relieved at longer distance. We note that the shift to higher energy of the $5 \tilde{\sigma}$ in the simulations is significantly larger than in the deconvolution of the experimental spectra. Lacking an absolute energy scale in the computations (emission energies are taken as differences between orbital energies) we refrain from drawing firm conclusions, but speculate that some of the intensity assigned as $\tilde{d}_{\pi}$ at 7 and $12 \mathrm{ps}$ time delay in the experiment might be $5 \tilde{\sigma}$ which would imply an even weaker bond to the substrate at these long delays. In the $\pi$-system we see 
qualitatively similar effects in experiment and simulation with strongly increased $1 \tilde{\pi}$ intensity as the $\pi$ and $\pi^{*}$ mixing becomes less at longer distances. In the calculated spectra we observe a near-complete loss of $\tilde{d}_{\pi}$ at the longer distance while in the experiment some intensity remains (with the caveat that the $5 \tilde{\sigma}$ position here is somewhat uncertain). Overall, however, we find good qualitative agreement between the computed and experimental spectra lending further credibility to the picture of a weakened but not completely broken bond to the substrate.

\section{Summary}

We have performed DFT calculations of $\mathrm{CO}$ desorption from $\mathrm{Ru}(0001)$ which predict a weakly bound state prior to desorption if non-local correlation is employed. Including the elevated temperature in the experiment through the PMF when modeling the desorption path, we find that an entropic barrier develops separating the chemisorbed and precursor state; at temperatures relevant for pump-probe experiments, this barrier becomes significant. Pump-probe XAS/XES indicate population of a precursor state to desorption upon laser-excitation of the system. Simulating spectra along the path of desorption supports the picture of a vdW stabilized transient state arising from laser-induced heating of the $\mathrm{CO} / \mathrm{Ru}$ system.

\section{Acknowledgment}

This work is supported by the Department of Energy, Office of Basic Energy Sciences, Division of Materials Sciences and Engineering, under contract DE-AC02-76SF00515, the US Department of Energy, Basic Energy Science through the SUNCAT Center for Interface Science and Catalysis, the Swedish National Research Council, the Danish Center for Scientific Computing, the Volkswagen Stiftung, the Alexander von Humboldt Foundation and the Lundbeck Foundation. The spectrum calculations were performed on resources provided by the Swedish National Infrastructure for Computing (SNIC) at the HPC2N center. Portions of this research were carried out on the SXR Instrument at the Linac Coherent Light Source (LCLS), a division of SLAC National Accelerator Laboratory and an Office of Science user facility operated by Stanford University for the U.S. Department of Energy. The SXR Instrument is funded by a consortium whose 
membership includes the LCLS, Stanford University through the Stanford Institute for Materials Energy Sciences (SIMES), Lawrence Berkeley National Laboratory (LBNL), University of Hamburg through the BMBF priority program FSP 301, and the Center for Free Electron Laser Science (CFEL). 


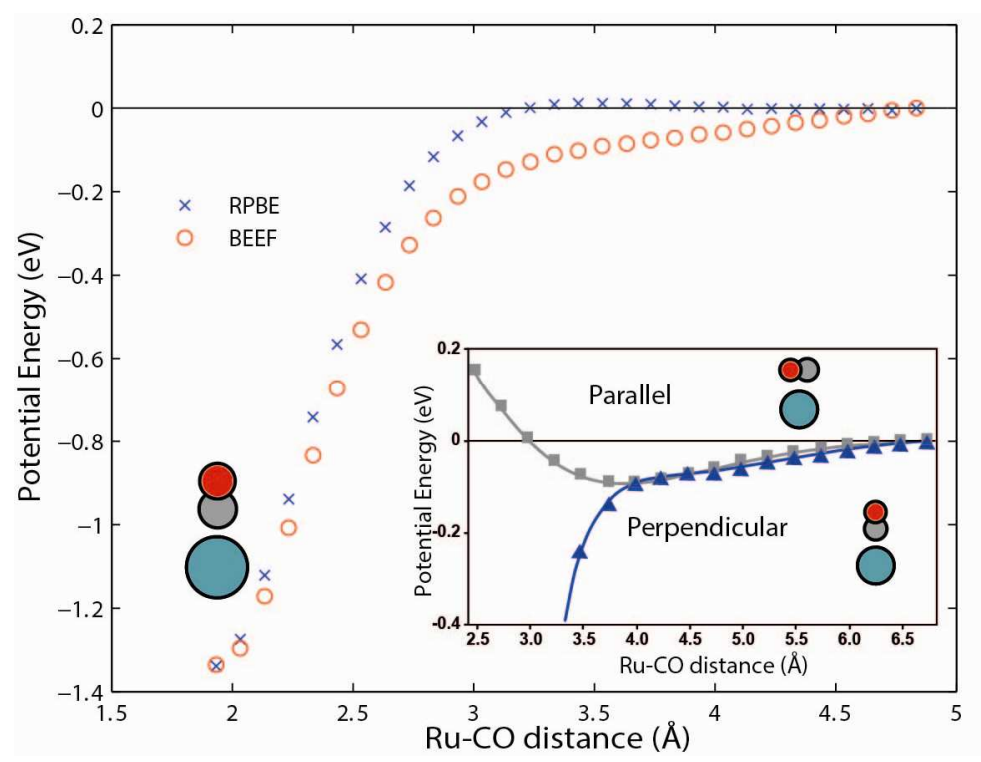

Fig. 1. Minimum energy path (MEP) of the $\mathrm{CO}$ desorption from the $\mathrm{Ru}(0001)$ surface using the RPBE (blue x-marks) and BEEF-vdW (red circles) exchange-correlation functionals. The potential energy is plotted as a function of the $\mathrm{C}-\mathrm{Ru}$ distance. The inset shows a region of the desorption paths for $\mathrm{CO}$ perpendicular (blue triangles) and parallel (grey squares) to the surface calculated using the BEEF-vdW functional. At $\sim 3.5 \AA$ (CO center of mass distance to the Ru surface), the attractive vdW plateau develops and the potential energy becomes independent of the $\mathrm{CO}$ orientation relative the surface. 


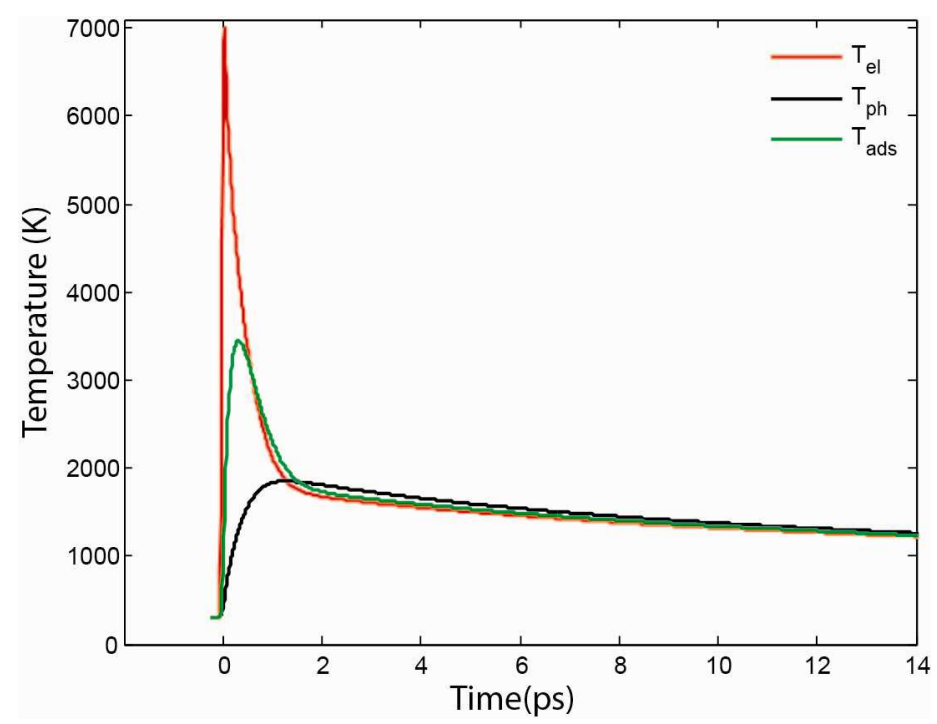

Fig. 2. Modeled time-evolution of electron- $\left(T_{\mathrm{el}}\right.$, red) and phonon- $\left(\mathrm{T}_{\mathrm{ph}}\right.$, black) temperatures of the $\mathrm{Ru}(0001)$ surface following absorption of a $140 \mathrm{~J} / \mathrm{m}^{2}$ laser pulse with a pulse length of $50 \mathrm{fs}(\mathrm{FWHM})$ and wavelength $400 \mathrm{~nm}$ at a base temperature of $300 \mathrm{~K}$. In addition, the adsorbate temperature of $\mathrm{CO}\left(\mathrm{T}_{\mathrm{ads}}\right.$, green) is plotted, see main text for calculation procedure. 
A
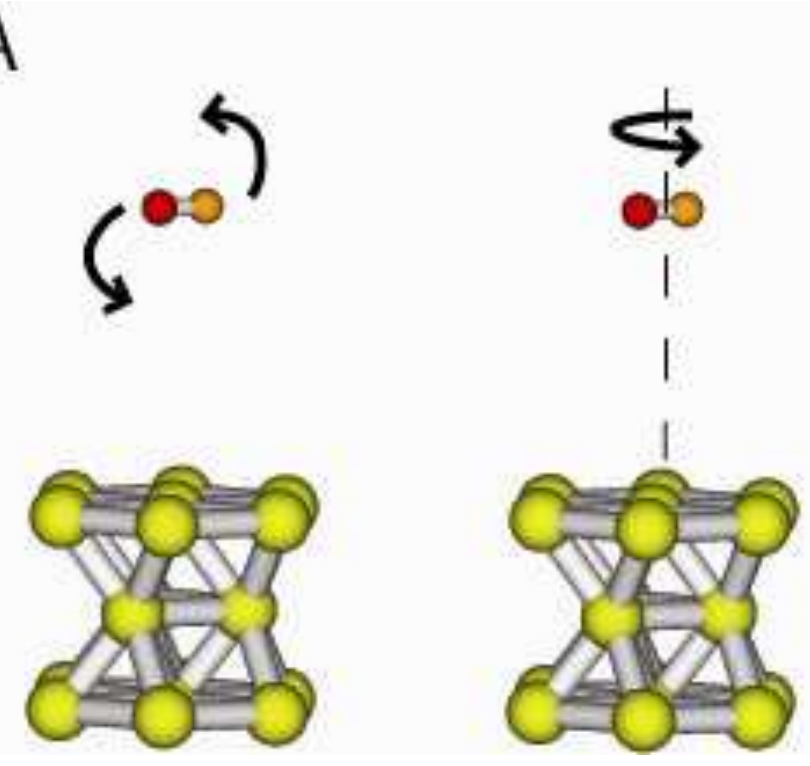

Fig. 3. Illustration of the rotational (A) cartwheel and (B) helicopter modes used in constructing the potential of mean force. 


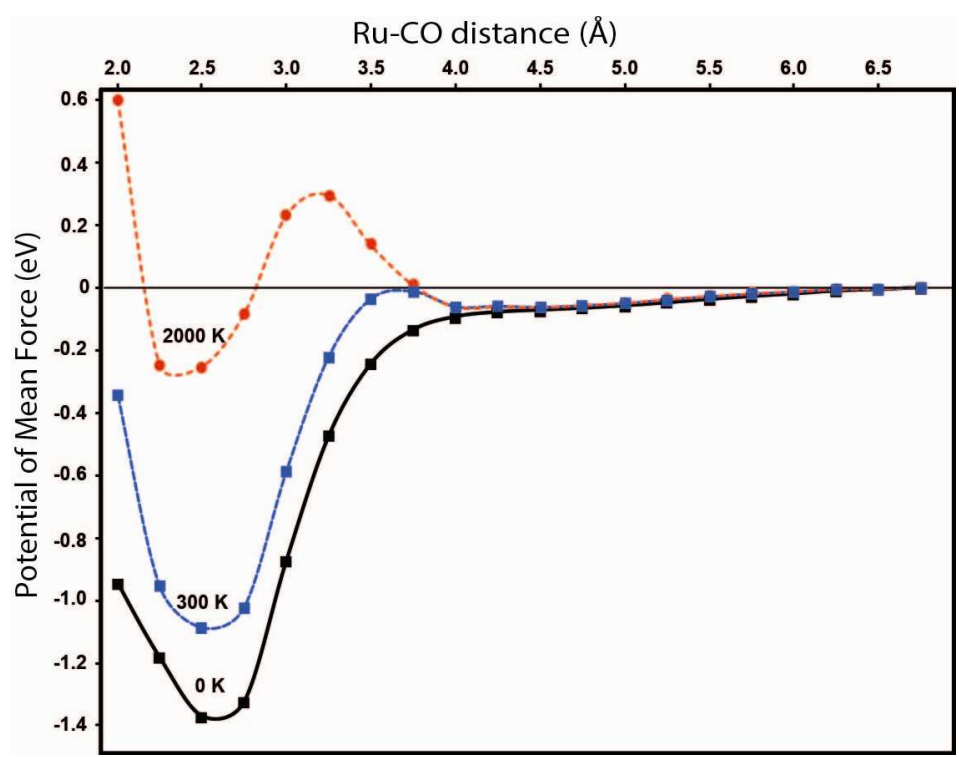

Fig. 4. The potential of mean force for $\mathrm{CO}$ adsorption/desorption on $\mathrm{Ru}(0001)$ at $0 \mathrm{~K}$ (MEP), 300 and $2000 \mathrm{~K}$ (black, blue and red curves, respectively). 

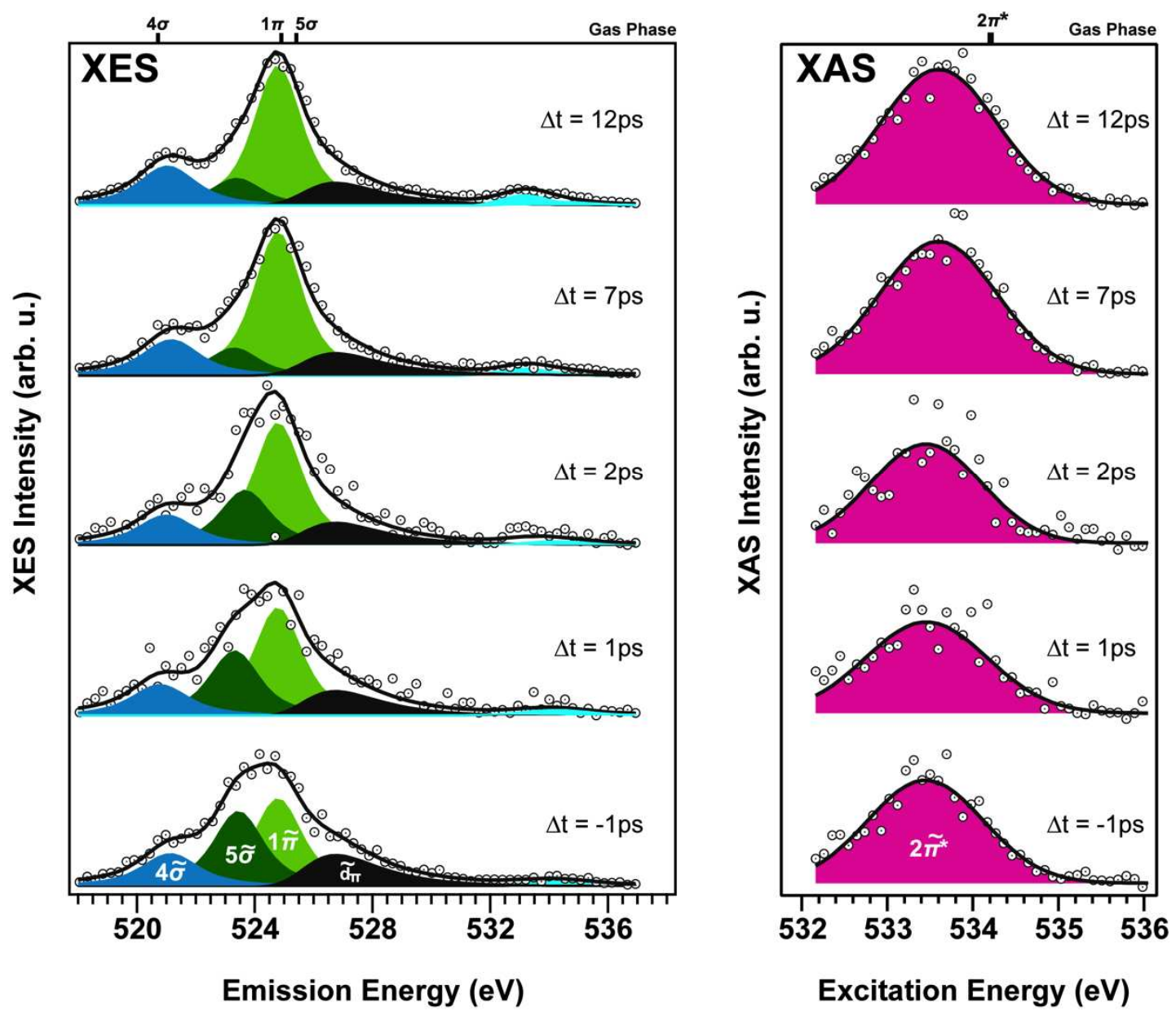

Fig. 5. Oxygen K-edge XE (left graph) spectra (markers) of $\mathrm{CO} / \mathrm{Ru}(0001)$ and relative fits (solid lines) measured at selected pump-probe delays $(\Delta \mathrm{t})$ presented in the paper. For each XE spectrum the peak deconvolution resulting from the fit is also shown as filled areas. The spectra were fitted with three peaks of Voigt lineshape for the $1 \tilde{\pi}$ (light green), $5 \tilde{\sigma}$ (dark green), $4 \tilde{\sigma}$ (blue) orbitals and an asymmetric Gaussian for the $\tilde{d}_{\pi}$ states (black). The O $1 s \rightarrow 2 \tilde{\pi}^{*}$ resonance in the XA data was fitted with a Gaussian function (purple). 

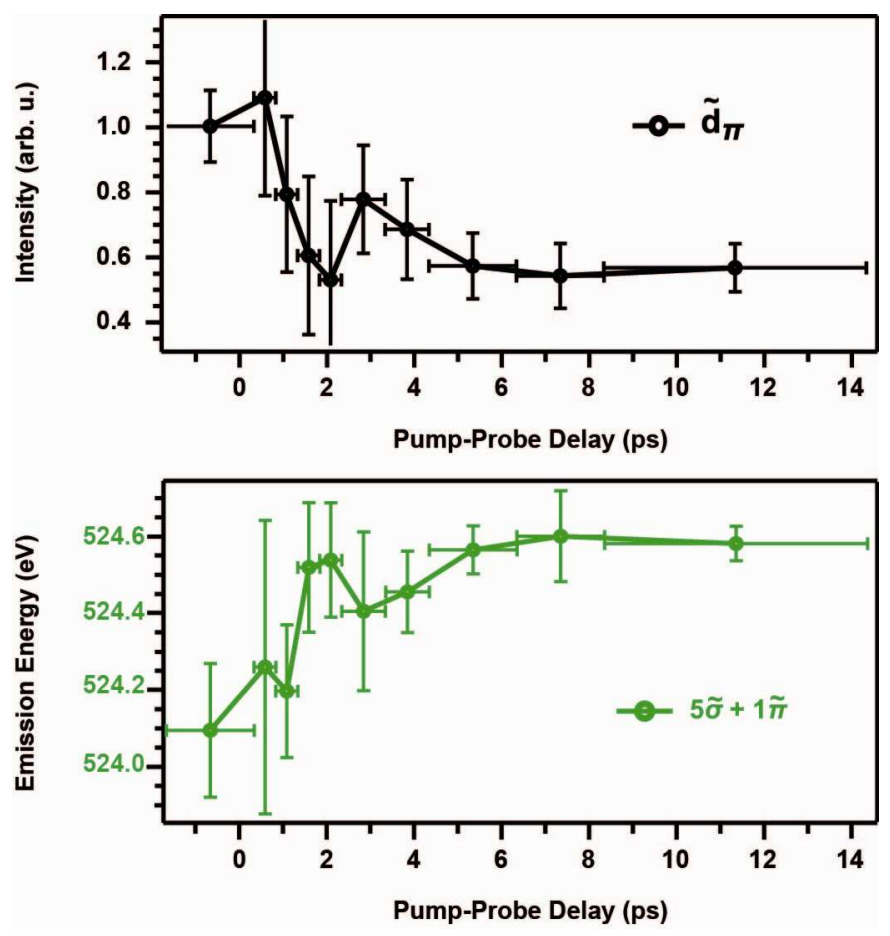

Fig. 6. XES (shown in Fig. 5) peak changes as a function of pump-probe delay: the $\tilde{d}_{\pi}$ intensity (upper panel) and emission energy of the center of mass of the $1 \tilde{\pi}$ and $5 \tilde{\sigma}$ components as functions of pump-probe delay (bottom panel). 


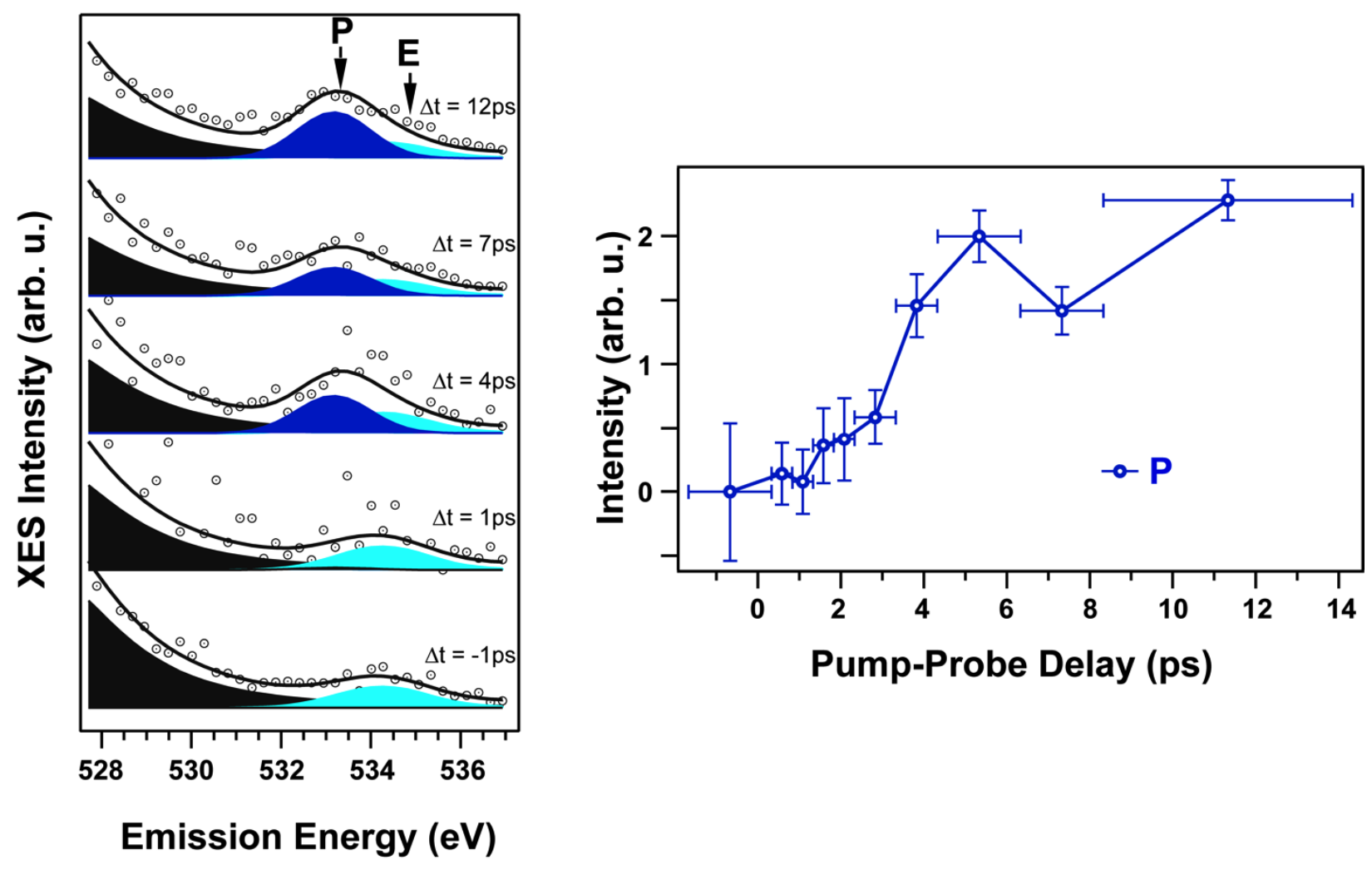

Fig. 7. Oxygen K-edge XE spectra and fits (left) in the emission energy region corresponding to elastically scattered photons (between $532 \mathrm{eV}$ and $537 \mathrm{eV}$ ) measured at selected pump-probe delays $(\Delta \mathrm{t})$. In the spectrum at $\Delta \mathrm{t}=-1 \mathrm{ps}$, peak $\mathrm{E}$ (at $534.3 \mathrm{eV}$, FWHM $2.2 \mathrm{eV}$ ) is the contribution from the elastic scattering. Peak P (at $533.2 \mathrm{eV}$, FWHM $1.6 \mathrm{eV}$ ), which appears at later delays, is the participator contribution and its intensity increases as a function of pump-probe delay (right panel). This contribution, which has been well characterized in gas phase measurements [48], is an indication of the development of a population of molecules less strongly bound to the surface. 

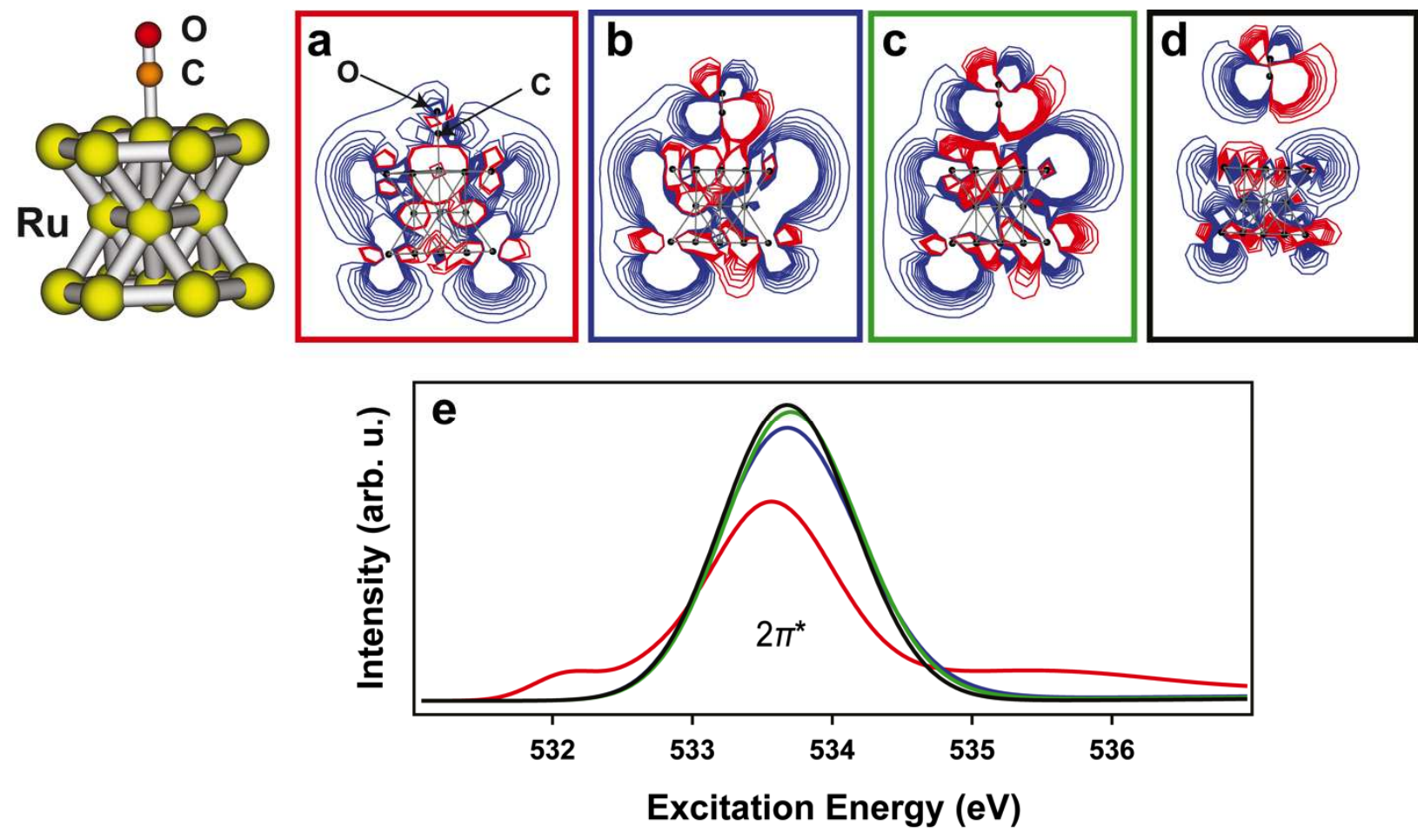

Fig. 8. (a-d) Iso-amplitude plots of wave functions of the core excited $2 \pi^{*}$ state calculated for $\mathrm{CO}$ on a 17-atom cluster model of the $\mathrm{Ru}(0001)$ surface sampling the potential energy surface from Figs. 1 and 4; blue indicates positive amplitude and red indicates negative amplitude in the wave function. (a) Equilibrium structure for ontop CO. Center-of-mass (COM) distance $2.601 \AA$. (b) COM distance $4.0 \AA$ (close to the inner turning point of the precursor well). (c) COM distance $4.5 \AA$ (in the precursor well). (d) COM distance $6.75 \AA$ (at the outer turning point of the precursor well). (e) Computed XA spectra of the different structures, color-coded according to the frames in (a-d). 


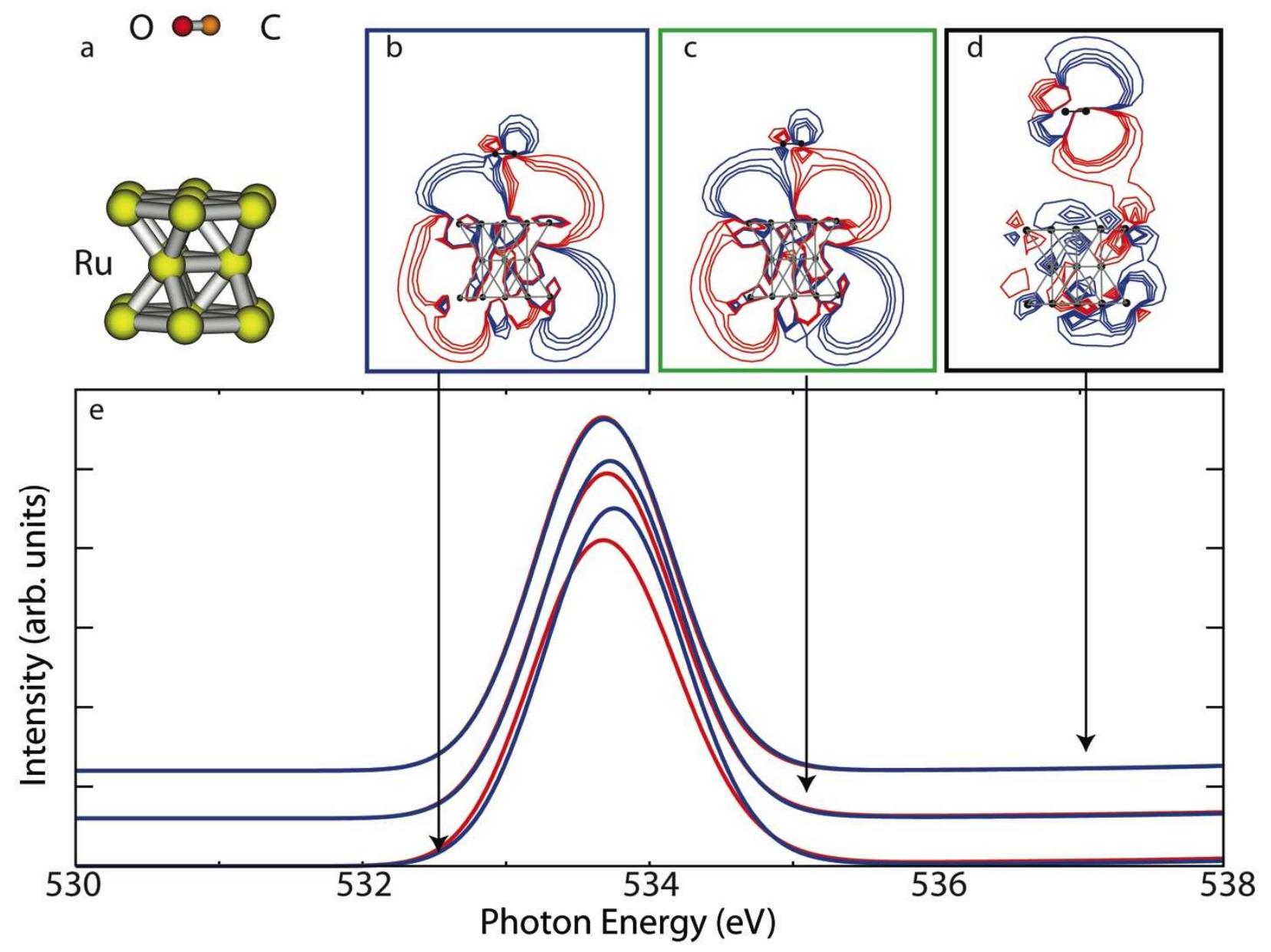

Fig. 9. Computed XA spectra and outer part of the $2 \pi^{*}$ core-excited state for CO rotated into a horizontal geometry (a) in the precursor state; explanation as in Fig. 8. (b-d) Spatial extent of the computed $2 \pi^{*}$ core-excited state in the precursor state at the same center-of-mass (COM) distances as in figure 8. (e) Comparison of computed XA spectra at the different COM distances (indicated by arrows) between vertical (red) and horizontal (blue) $\mathrm{CO}$ in the precursor state. 


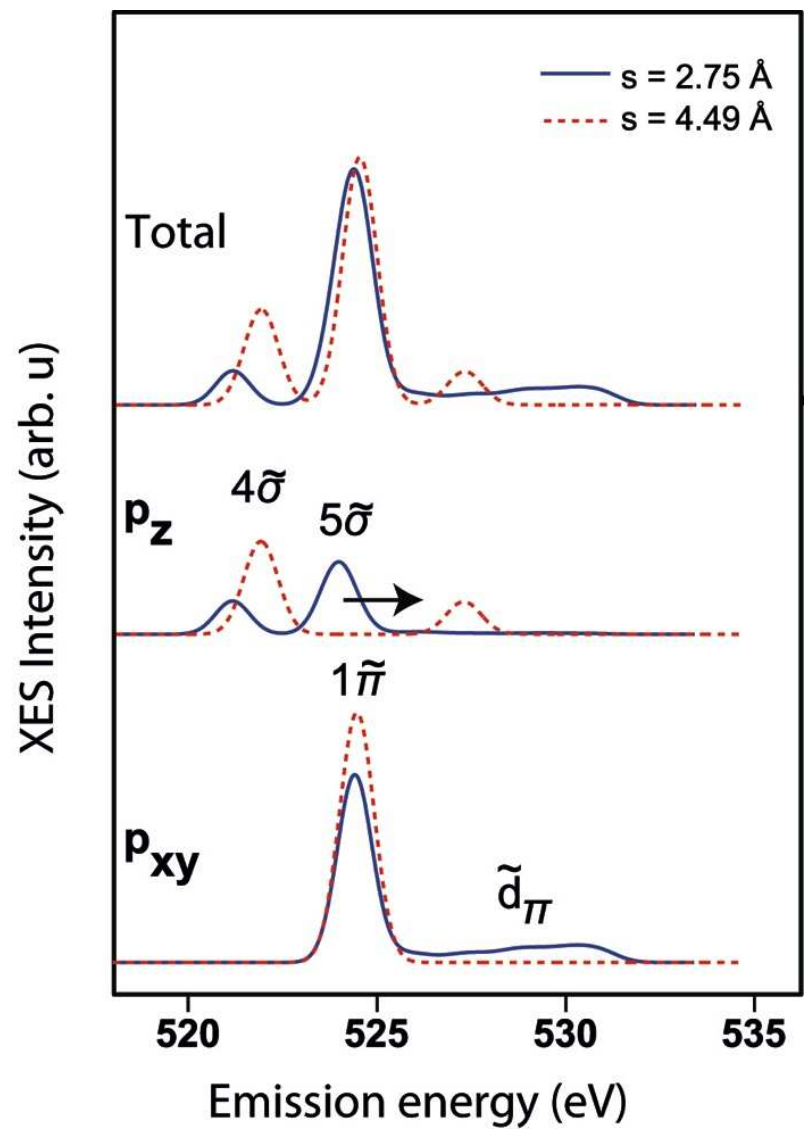

Fig. 10. Computed (non-resonant) $\mathrm{XE}$ spectra of $\mathrm{CO}$ on $\mathrm{Ru}(0001)$ at distances corresponding to the minimum in the chemisorption state (blue) and at the precursor state (red and dashed) in Fig. 4. The spectra from top to bottom are the total spectrum and resolved in pz and pxy components. The distance, $s$, from the Ru surface to the center of mass of the $\mathrm{CO}$ molecule is $2.75 \AA$ in the chemisorbed state and $4.49 \AA$ in the precursor well. The calculated spectra are shifted so that the energy position of the $1 \tilde{\pi}$ state is aligned with that of the corresponding experimental spectrum. 


\section{References}

[1] A. Föhlisch, M. Nyberg, P. Bennich, L. Triguero, J. Hasselström, O. Karis, L.G.M. Pettersson, A. Nilsson, J. Chem. Phys. 112 (2000) 1946.

[2] A. Föhlisch, M. Nyberg, J. Hasselström, O. Karis, L.G.M. Pettersson, A. Nilsson, Phys. Rev. Lett 85 (2000) 3309.

[3] J. Gladh, H. Öberg, J. Li, M.P. Ljungberg, A. Matsuda, H. Ogasawara, A. Nilsson, L.G.M. Pettersson, H. Öström, J. Chem. Phys. 136 (2012) 034702

[4] A. Nilsson, L.G.M. Pettersson, Surf. Sci. Rep. 55 (2004) 49-167.

[5] P. Bennich, T. Wiell, O. Karis, M. Weinelt, N. Wassdahl, A. Nilsson, M. Nyberg, L.G.M. Pettersson, J. Stöhr, M. Samant, Phys. Rev. B 57 (1998) 9274.

[6] G. Blyholder, J. Phys. Chem. 68 (1964) 2772.

[7] P.S. Bagus, C.J. Nelin, C.W. Bauschlicher, Phys. Rev. B 28 (1983) 5423.

[8] P.S. Bagus, K. Hermann, Phys. Rev. B 33 (1986) 2987.

[9] J. Rogozik, V. Dose, K.C. Prince, A.M. Bradshaw, P.S. Bagus, K. Hermann, P. Avouris, Phys. Rev. B 32 (1985) 4296.

[10] M. Nyberg, L. Triguero, A. Bassan, L.G.M. Pettersson, A. Föhlisch, A. Nilsson, J. Mol. Struc. (THEOCHEM) 762 (2006) 123-132

[11] C.W. Bauschlicher, L.G.M. Pettersson, P.E.M. Siegbahn, J. Chem. Phys. 87 (1987) 2129.

[12] P.J. Feibelman, B. Hammer, J.K. Nørskov, F. Wagner, M. Scheffler, R. Stumpf, R. Watwe, J. Dumesic, J. Phys. Chem. B 105 (2001) 4018.

[13] F. Delbecq, P. Sautet, Phys. Rev. B 59 (1999) 5142.

[14] G. Kresse, A. Gil, P. Sautet, Phys. Rev. B 68 (2003) 073401

[15] R.A. Olsen, P.H.T. Philipsen, E.J. Baerends, J. Chem. Phys. 119 (2003) 4522

[16] N. Dimakis, T. Mion, E.S. Smotkin, J. Phys. Chem. C 116 (2012) 21447-21454.

[17] L.G.M. Pettersson, A. Nilsson, Topics in Catalysis 57 (2014) 2-13.

[18] M. Dell'Angela, et al., Science 339 (2013) 1302.

[19] M. Beye, et al., Phys. Rev. Lett. 110 (2013) 186101.

[20] J. Wellendorff, K.T. Lundgaard, A. Møgelhøj, V. Petzold, D.D. Landis, J.K. Nørskov, T. Bligaard, K.W. Jacobsen, Phys. Rev. B 85 (2012) 235149.

[21] J. Enkovaara, C. Rostgaard, J.J. Mortensen, J. Phys.: Condens. Matter 22 (2010) 253202.

[22] J.J. Mortensen, L.B. Hansen, K.W. Jacobsen, Phys. Rev. B 71 (2005) 035109.

[23] B. Hammer, L.B. Hansen, J.K. Nørskov, Phys. Rev. B 59 (1999) 7413.

[24] M.P. Ljungberg, J.J. Mortensen, L.G.M. Pettersson, J. El. Spec. Rel. Phenom. 184 (2011) 427.

[25] A. Föhlisch, J. Hasselström, P. Bennich, N. Wassdahl, O. Karis, A. Nilsson, L. Triguero, M. Nyberg, L.G.M. Pettersson, Phys. Rev. B 61 (2000) 16229-16240.

[26] K. Hermann, et al., deMon Software: Stockholm-Berlin, 2011.

[27] W. Kutzelnigg, U. Fleischer, M. Schindler, NMR-Basic Principles and Progress, Springer Verlag, Heidelberg, 1990.

[28] L. Triguero, L.G.M. Pettersson, H. Ågren, Phys. Rev. B 58 (1998) 8097.

[29] M. Leetmaa, M.P. Ljungberg, A.P. Lyubartsev, A. Nilsson, L.G.M. Pettersson, J. El. Spec. Rel. Phen. 177 (2010) 135-157

[30] A.D. Becke, Physical Review A 38 (1988) 3098-3100. 
[31] J.P. Perdew, Physical Review B 33 (1986) 8822-8824.

[32] O. Takahashi, L.G.M. Pettersson, J. Chem. Phys. 121 (2004) 10339-10345.

[33] P. Heimann, et al., Rev. Sci. Instrum. 82 (2011) 093104.

[34] W.F. Schlotter, et al., Rev. Sci. Instrum. 83 (2012) 043107.

[35] T. Katayama, et al., J. Electron Spectrosc. Relat. Phenom. 187 (2013) 9-14.

[36] Emma P., et al., Nat. Photon. 4 (2010) 641-647.

[37] S.I. Anisimov, B.L. Kapeliovich, T.L. Perel'man, Sov. Phys. JETP 39 (1974) 375

[38] M. Lisowski, P.A. Loukakos, U. Bovensiepen, J. Stähler, C. Gahl, M. Wolf, App. Phys. A 78 (2004) 165-176.

[39] S. Funk, M. Bonn, D.N. Denzler, C. Hess, M. Wolf, G. Ertl, J. Chem. Phys. 112 (2000) 9888.

[40] M. Lisowski, P.A. Loukakos, U. Bovensiepen, J. Stähler, C. Gahl, M. Wolf, Appl. Phys. A 78 (2004) 165-176.

[41] J.A. Prybyla, H.W.K. Tom, G.D. Aumiller, Phys. Rev. Lett. 68 (1992) 503.

[42] P. Szymanski, A.L. Harris, N. Camillone III, J. Phys. Chem. A 111 (2007) 12524.

[43] J. Gladh, T. Hansson, H. Öström, Surf. Sci. 615 (2013) 65-71.

[44] D.J. Doren, J.C. Tully, Langmuir 4 (1988) 256-268.

[45] D.J. Doren, J.C. Tully, The Journal of Chemical Physics 94 (1991) 8428-8440.

[46] H. Xin, et al., Phys. Rev. Lett. (2015) submitted.

[47] A. Nilsson, L.G.M. Pettersson, Surf. Sci. Rep. 55 (2004) 49-167.

[48] P. Skytt, P. Glans, K. Gunnelin, J.H. Guo, J. Nordgren, Y. Luo, H. Ågren, Phys. Rev. A 55 (1997) 812.

[49] C. Keller, M. Stichler, G. Comelli, F. Esch, S. Lizzit, W. Wurth, D. Menzel, Phys. Rev. Lett. 80 (1998) 1774-1777.

[50] F. Gel'mukhanov, H. Ågren, Phys. Reps. 312 (1999) 87-330. 